\title{
ROBUST MULTI-USER OPPORTUNISTIC BEAMFORMING FOR SPARSE NETWORKS
}

\author{
Marios Kountouris \\ France Telecom R\&D \\ Issy-les-Moulineaux, France \\ marios.kountouris@francetelecom.com
}

\author{
David Gesbert
}

\author{
Eurecom Institute \\ Sophia-Antipolis, France \\ david.gesbert@eurecom.fr
}

\begin{abstract}
A scheme exploiting reduced feedback for the purpose of opportunistic multi-user beamforming is proposed. The scheme builds on recent promising advances realized in the area of multi-user downlink precoding and scheduling based on partial transmitter channel state information (CSIT) using random beamforming-based SDMA. Although random precoding followed by SDMA scheduling is optimal within the set of unitary precoders, it is only so in the asymptotic number of users $K$. For -practically relevant- sparse networks (i.e. with low to moderate number of users) random beamforming SDMA yields severely degraded performance. In this work we present a scheme allowing to restore robustness with respect to cell sparsity. The core idea here is to preserve the low complexity low feedback advantage of random opportunistic beamforming in selecting a target group of users, while much more efficient beamforming schemes can be used to serve the group of users once it has been identified. We propose different designs, optimal and suboptimal, based upon variable levels of feedback requirement. We show substantial gain over opportunistic beamforming for a range of $K$.
\end{abstract}

\section{INTRODUCTION}

The design of MIMO transmit/receive physical layer schemes which lend themselves well to integration with efficient protocols for resource allocation at medium access control (MAC) layer represent a critical open area for current research. In this framework of cross layer design, two problems deserve particular attention: 1- the joint design of antenna combining schemes at the transmitter together with scheduling protocols, 2-resolution of the problem above under constraint of reasonably low feedback of CSIT and complexity.

In several recent papers, it was recognized that the choice of a proper multiple access technique (TDMA versus SDMA) combined with antenna combining technique (Space time coding versus beamforming) hinges heavily on the nature and quality of the feedback channel bringing channel-related information (CSIT) to the transmitter/scheduler [1]. For narrow and/or error-prone feedback channels, space-time coding combined with multi-user diversity TDMA-like scheduling algorithms [3] seems a reasonable option [2]. However for a system with reasonably accurate and/or complete CSIT it is beneficial to exploit the spatial multiplexing capability of transmit antennas to several users at once rather than trying to maximize the reliability/diversity of a single user link. To realize this, optimal schemes based on the dirty paper coding approach have been proposed [4], as well as suboptimal greedy techniques for solving the precoding and multi-user power allocation problem [5]. Unfortunately the applicability of such schemes is limited due to 1-computational complexity and 2-the need for full CSIT across all active cell users which may lead to prohibitive feedback requirements in FDD systems or lack of robustness to CSIT errors in TDD setups with mobility.

Interestingly, in [6] a low-feedback scheme was proposed exploiting a unitary precoder (multi-user beamforming matrix) together with an optimally matching selected set of $N_{t}$ spatially multiplexed users per slot, where $N_{t}$ is the number of transmit antennas at the base station. The idea of [6] builds on the concept of opportunistic beamforming as initially shown in [7], to the difference that it is extended to the multi-user multi-beam situation.

Random unitary precoding offers optimal scaling laws of capacity when the number of users is large and requires only little feedback from the users (in the form of individual SINRs). Unfortunately, this scheme is quickly degrading with decreasing number of users. Furthermore, this degradation is amplified when the number of transmit antennas increases. The reason is intuitive: As the number of active users decreases and $N_{t}$ increases, it becomes more and more unlikely that $N_{t}$ randomly generated, equipowered beams will match well the vector channels of any set of $N_{t}$ users in the cell. This is a major problem as traffic is normally bursty with frequent silent periods in data-access networks thus the scheduler may not count on a large number of simultaneously active users at all times.

In this paper we investigate a new class of random beamforming exhibiting robustness with respect to user sparsity while preserving low feedback and low complexity advan- 
tages compared over full CSIT-based schemes $[4,5]$. Previously we have proposed a method exploiting memory in temporal channel variations [8]. Here we present an independent approach requiring no special channel property.

We make the following key points: In channel-aware optimal and near-optimal schemes (e.g. $[4,5])$, the multiuser CSIT thus serves two distinct purposes 1- identify a group of $N$ selected users with mutually-good channel conditions (typically $N \leq N_{t}$ if linear beamforming is used), and 2-compute the beamforming vectors used to serve these $N$ users. However we remark that the bulk of the feedback load and complexity in such schemes is dominated by the need to process the full CSIT of all (say $K$ ) active users in view of the user group selection process. Based on this remark we make the following proposal and contributions:

- In this paper, a low complexity low feedback user group selection scheme is presented based on random unitary beamforming, reminiscent of [6].

- Given the pre-selected set of $N$ users, a second step exploiting linear beamforming is applied to serve the selected users. The second step beamforming matrix may require variable levels of additional CSIT feedback to be computed, depending on design.

- We point out that even if optimal beamforming with full CSIT is chosen, it only involves $N \leq N_{t}<<$ $K$ users, thus marking a significant complexity and feedback gain over optimal techniques $[4,5]$.

- In general, the second step beamformer will be different from the random beamformer used in [6]. In particular, while we expect little gain over [6] for large $K$, we expect significant gain for sparse systems with low to moderate $K$ (compared with $N_{t}$ ) where the initial random beamformer may not provide satisfactory rate for all $N_{t}$ users.

- In one version of the proposed designs, we propose a simple power allocation scheme across the beams of the random beamformer showing substantial capacity improvement over [6] for realistic values of $K$. Both closed-form and iterative solutions are investigated and numerically simulated.

\section{NETWORK AND SIGNAL MODEL}

We consider a multiple antenna broadcast (downlink) channel with $K$ users in which the transmitter (base station) is equipped with $N_{t}$ antennas, and each user terminal with $N_{r}$ antennas. The received signal $\mathbf{y}_{k}(t) \in \mathbb{C}^{N_{r} \times 1}$ at user $k$ at time slot $t$ is mathematically described as

$$
\mathbf{y}_{k}(t)=\mathbf{h}_{k} \mathbf{x}(t)+\mathbf{n}_{k}, k=1, \ldots, K
$$

where $\mathbf{x}(t) \in \mathbb{C}^{N_{t} \times 1}$ is the transmitted vector signal at time slot $t, \mathbf{h}_{k} \in \mathbb{C}^{N_{r} \times N_{t}}$ is the complex channel matrix, and $\mathbf{n}_{k} \in \mathbb{C}^{N_{r} \times 1}$ is the circularly symmetric complex Gaussian noise at receiver $k$. We assume that the channel matrix $\mathbf{h}_{k}$ is perfectly known to the receiver, and that the elements of $\mathbf{h}_{k}$ and $\mathbf{n}_{k}$ have a zero mean and unit variance complex Gaussian distribution. The transmitter is subject to a power constraint $P, \operatorname{trace}\left(\left\{\mathbf{x x}^{H}\right\}\right) \leq P$. Due to the noise variance normalization, $P$ takes the meaning of maximum transmit signal-to-noise ratio (SNR). We let $\mathbf{H} \in \mathbb{C}^{N_{r} K \times N_{t}}$ refer to the concatenation of all channels, where $\mathbf{H}=\left[\mathbf{h}_{1}^{T}, \ldots, \mathbf{h}_{K}^{T}\right]^{T}$. In the following sections, for simplicity, we assume $N_{r}=1$.

\section{CAPACITY OF MULTI-USER MIMO BROADCAST CHANNELS}

If full channel knowledge is available at the transmitter for all $K$ users, the sum rate is equal to (using the duality[9])

$C_{\text {sum }}=\mathbb{E}\left\{\max _{P_{1}, \ldots, P_{K}, \sum_{k} P_{k}=P} \log \operatorname{det}\left(\mathbf{I}+\sum_{\mathbf{k}=\mathbf{1}}^{\mathbf{K}} \mathbf{h}_{\mathbf{k}}^{*} \mathbf{P}_{\mathbf{k}} \mathbf{h}_{\mathbf{k}}\right)\right\}$

where $\mathbf{h}_{k}$ is $1 \times N_{t}$ channel matrix with i.i.d. $\mathbb{C N}(0,1)$ distributions, $P_{k}$ is the power allocated to user $k$. The sum rate capacity of MIMO BC channel has been examined by several authors [4][9], and it was shown that the capacityachieving strategy in multi-user MIMO downlink is dirty paper coding (DPC). As DPC is difficult to implement in practice, random linear beamforming with unitary precoding matrices was also investigated in order reduce complexity and feedback. In [10], it was shown that for fixed total average transmit power and $N_{t}$, the sum rate for DPC and beamforming scales like $N_{t} \log \log K N_{r}$. Furthermore, as the random beamforming capacity is a lower bound for the capacity of optimum beamforming, the latter should also have the same scaling laws.

\section{REVIEW OF RANDOM MULTI-USER BEAMFORMING}

Here we assume temporarily $N=N_{t}$. In [6], the unitary beamforming matrix $\mathbf{Q}$ is drawn randomly in an effort to reduce feedback and complexity requirements. The $N_{t}$ columns of $\mathbf{Q}$ are interpreted as random beams. Over each beam the user with the highest signal-to-interference-plusnoise ratios (SINR) on that beam is served. The random beams are generated independently from one time slot to the other. Assuming $N_{r}=1$, a $N_{t} \times N_{t}$ unitary matrix $\mathbf{Q}$ is generated according to an isotropic distribution. At time 
slot $t$ the transmitted signal is

$$
\mathbf{x}(t)=\sum_{m=1}^{N_{t}} \mathbf{q}_{m}(t) s_{m}(t)
$$

where $s_{m}(t)$ is the m-th transmit symbol at time slot $t$ and $\mathbf{q}_{m} \in \mathbb{C}^{N_{t} \times 1}$ are random orthonormal vectors (beams) for $m=1, \ldots, N_{t}$ (columns of $\mathbf{Q}$ ). The received signal at the $k$-th receiver is

$$
y_{k}=\sum_{m=1}^{N_{t}} \mathbf{h}_{k} \mathbf{q}_{m} s_{m}+\mathbf{n}_{k}, k=1, \ldots, K
$$

The SINRs of user $k$ on beam $m$ is

$$
S I N R_{k, m}=\frac{\gamma_{k m}}{\sigma^{2}+\sum_{j \neq m} \gamma_{k j}}
$$

where $\gamma_{i j}=\left|\mathbf{h}_{i} \mathbf{q}_{j}\right|^{2}$, and $\sigma^{2}=N_{t} / P$. Each user feeds back its maximum SINR and the index $m$ of the beam for which its SINR is maximized. In turn, for each beam $\mathbf{q}_{m}$ the transmitter assigns the beam to the user with the highest corresponding SINR, i.e. $\arg _{k} \max S I N R_{k, m}$. The sum rate of the above scheme is given by [6],

$$
C_{\text {sum }} \approx \mathbb{E}\left\{\sum_{m=1}^{N_{t}} \log _{2}\left(1+\max _{1 \leq k \leq K} S I N R_{k, m}\right)\right\}
$$

\section{ROBUST RANDOM BEAMFORMING}

Let us denote $\mathbf{I}^{(r)}$ the scheduling vector containing the index of $N_{t}$ users selected via the scheme above. For sparse networks, the number of users $K$ is not high enough to be confident that all $N_{t}$ users in $\mathbf{I}^{(r)}$ enjoy a reasonable SINR because the selected users may not be fully separable under unitary beamforming $\mathbf{Q}$, though, as we point out, this group is likely to exhibit mutually-good channel conditions, relative to the rest of the users, since it is the best group under $\mathrm{Q}$ at least. To improve performance we propose to augment the random beamforming step with a second step where additional CSIT feedback is provided, yet only involving the $N_{t}$ pre-selected users. We examine various level of feedback and corresponding optimal beamforming designs. We show that significant gains can be achieved with minimal feedback.

\subsection{Full CSIT knowledge for the pre-selected $N_{t}$ users}

In this case, once the group is determined (in the form of $\mathbf{I}^{(r)}$ ), one requests full CSIT feedback for the $N_{t}$ selected users. Note that this results in an overall feedback requirement much inferior to that of $[4,5]$. In this case, for any set of transmission powers $\mathbf{p}=\left[P_{1}, \ldots, P_{K}\right]^{T}$, the linear detector that maximizes the SINR of each user is the beamforming matrix $\mathbf{W}$, computed according to:

$$
\mathbf{W}=\mathbf{H}\left[\mathbf{I}^{(\mathbf{r})}\right]\left(\sigma^{2} \mathbf{I}+\mathbf{H}\left[\mathbf{I}^{(\mathbf{r})}\right]^{\mathbf{H}} \mathbf{H}\left[\mathbf{I}^{(\mathbf{r})}\right]\right)^{-\mathbf{1}}
$$

Note that the optimal precoding matrix in the downlink is derived from the uplink MMSE beamformer and based on the uplink-downlink duality [11]. Using the random beamforming as a user selection scheme, a set of quasi-orthogonal is revealed to the transmitter that then proceeds to MMSE precoding. The suboptimality of the scheme depends on the sparsity of the system. The more users are in the cell, the more likely is to select an orthogonal user group at the first step. The performance of MMSE downlink precoder can be enhanced using power allocation. However, the solution to this optimization problem is not trivial, even if the duality is exploited [12].

\subsection{SIR knowledge for pre-selected $N_{t}$ users}

Here we assume only SIR type feedback is available. In particular, we assume the scheduler gains knowledge of $\gamma_{k m}$ for $k \in \mathbf{I}^{(r)}$. Based on this information we propose to design the beamforming matrix by applying a power allocation strategy across the beams of $\mathbf{Q}$. The SINR of user $k$ is given:

$$
S I N R_{k, m}=\frac{P_{m} \gamma_{k m}}{\sigma^{2}+\sum_{j \neq m} P_{j} \gamma_{k j}}
$$

The optimization problem of the transmit powers $\mathbf{p}$ that maximizes the throughput can be formulated as

$$
\begin{gathered}
\max _{\mathbf{p}} \sum_{k \in \mathbf{I}^{(r)}} \log \left(1+S I N R_{k, m}\right) \\
\text { subject to } \sum_{i=1}^{N_{t}} P_{i}=P
\end{gathered}
$$

In what follows we investigate a closed-form and an iterative solution to this problem.

\subsubsection{Closed-form solution for $N_{t}=2$}

The optimum power allocation scheme that maximizes the system throughput can be calculated analytically for a 2 beam system. The sum rate is given in terms of $P_{1} \in[0, P]$ by:

$$
J\left(P_{1}\right)=\log _{2}\left[\left(1+\frac{P_{1} \gamma_{11}}{\sigma^{2}+\left(P-P_{1}\right) \gamma_{12}}\right)\left(1+\frac{\left(P-P_{1}\right) \gamma_{22}}{\sigma^{2}+P_{1} \gamma_{21}}\right)\right]_{(9)}
$$

Lemma The optimum transmit power allocation strategy for a 2-beam system is given by:

$$
P_{1}^{o p t}=\operatorname{argmax}_{P_{1}=0, P,\left(-B \pm \sqrt{B^{2}-4 A \Gamma}\right) / 2 A} J\left(P_{1}\right)
$$




$$
P_{2}^{o p t}=P-P_{1}^{o p t}
$$

where $P_{1} \in[0, P]$ and

$$
\begin{gathered}
A=\gamma_{11} \gamma_{21}\left(P \gamma_{12}+\sigma^{2}\right)\left(\gamma_{21}-\gamma_{22}\right)+ \\
+\gamma_{22} \gamma_{12}\left(\gamma_{11}-\gamma_{12}\right)\left(P \gamma_{21}+\sigma^{2}\right) \\
B=\gamma_{11}\left(P \gamma_{12}+\sigma^{2}\right)\left(P \gamma_{21} \gamma_{22}+2 \gamma_{21} \sigma^{2}-\gamma_{22} \sigma^{2}\right)+ \\
+\gamma_{22}\left(P \gamma_{12}+\sigma^{2}\right)\left(P \gamma_{21}+\sigma^{2}\right)\left(2 \gamma_{12}-\gamma_{11}\right) \\
\Gamma=\gamma_{11} \sigma^{2}\left(P \gamma_{12}+\sigma^{2}\right)\left(P \gamma_{22}+\sigma^{2}\right) \\
-\gamma_{22}\left(P \gamma_{12}+\sigma^{2}\right)^{2}\left(P \gamma_{21}+\sigma^{2}\right)
\end{gathered}
$$

Proof As the objective function (9) is not always concave with respect to $P_{1}$, the $P_{1}$ that maximizes $J\left(P_{1}\right)$ is either the boundary points $\left(P_{1}=0\right.$ and $P_{1}=P$, greedy allocation) or the solutions corresponding to $\partial J / \partial P_{1}=0$. Taking the derivative of the objective function and setting $\partial J / \partial P_{1}=$ 0 , we have that the possible values of $P_{1}$ that maximize the throughput are the real-value roots of the second-order polynomial $A P_{1}^{2}+B P_{1}+\Gamma=0$ that satisfy the constraint $P_{1} \in[0, P]$. Hence, the optimum $P_{1}^{o p t}$ is the value among the boundary points and the roots of the polynomial that maximizes the objective function $J\left(P_{1}\right)$.

\subsubsection{Iterative solution for $N_{t}>2$}

In order to generalize the above mentioned power allocation scheme with partial CSIT, we propose an algorithm inspired by the iterative multi-user water-filling [14]. The intuition behind is that at every step, $\gamma_{k m} /\left(\sigma^{2}+\sum_{j \neq m} P_{j} \gamma_{k j}\right)$ is kept fixed and treated as noise. Given a sum power constraint, the problem is similar to multi-user water-filling and thus, all transmit powers $P_{m}$ assigned to beams can be calculated simultaneously so as to maintain a constant waterlevel. Let $\mathbf{p}^{(\mathbf{0})}=\mathbf{0}$ be the initial point. The steps of the algorithm are as follows:

$$
\begin{aligned}
& \overline{\text { Proposed Iterative Power Allocation Algorithm }} \\
& \text { For } n=1,2, \ldots \text { repeat } \\
& \text { Step 1 Calculate } \lambda_{k}=\frac{\gamma_{k m}}{\sigma^{2}+\sum_{j \neq m} P_{j}^{(n-1)} \gamma_{k j}}, \text { for } k \in \mathbf{I}^{(r)}
\end{aligned}
$$

Step 2 Let $\mathbf{p}^{(\mathbf{n})}$ be the power allocation solution of: $\max _{\mathbf{p}} \sum_{k} \log \left(1+P_{m} \lambda_{k}\right)$, subject to $\sum_{m} P_{m} \leq P$ yielding $P_{m}^{(n)}=\left[\mu-1 / \lambda_{k}\right]_{+}$, with $\sum_{k}\left[\mu-1 / \lambda_{k}\right]_{+}=P$ where $(x)_{+}=\max (0, x)$ and $\mu$ is the water-filled 'level'. In every step, the algorithm computes iteratively the optimal beam power allocation to increase sum capacity and converges to a limit value greater or equal to the sum-rate of equal power allocation.

\subsection{SINR knowledge for the pre-selected $N_{t}$ users}

Here we assume the scheduler has only access to the same information as what is available in [6], namely $S I N R_{k, m}$. However we further exploit this information in view of rendering the beamformer robust with respect to cases where not all $N_{t}$ users can be served satisfactorily simultaneously. We propose a strategy, coinded beam-on beam-off (BOBO), which can be viewed as coarser version of the power allocation earlier described. In the interest of space, we present the scheme for $N_{t}=2$. Generalization and details are revealed in [12]. In the BOBO scheme a policy is used by which certain beams are allocated full power while others get zero power, depending on the values of $S I N R_{k, m}$. Thus, in the $N_{t}=2$ case, we allocate power either 1equally as in [6], or 2- greedily to one particular beam. We investigate the proposed policy: let $\vartheta=S I N R_{\min } / S I N R_{\max }$. Let $f$ be a threshold with $0<f<1$ (see below for examples). When $\vartheta<f$ greedy allocation is applied in favor of the best beam (SDMA falls back to TDMA). In the opposite case, equal power is allocated.

\section{NUMERICAL EVALUATION}

We consider a block fading channel where the fading $\mathbf{h}_{k}(t)$ are i.i.d. among users and for different antennas. The plots are obtained through Monte-Carlo simulations and ergodic capacity is considered. In Fig. 1, we compare the sum rate performance of various downlink MISO strategies. As expected, the MMSE precoder applied to a set of quasiorthogonal users outperforms significantly the random unitary beamforming. The performance gain of $1.7 \mathrm{bps} / \mathrm{Hz}$ of MMSE beamformer can be further increased if optimal power allocation is used. In Fig. 2, the sum rate of the closed-form solution and iterative power allocation algorithm are plotted for $N_{t}=2, \mathrm{SNR}=0 \mathrm{~dB}$ and $N_{t}=4$, $\mathrm{SNR}=5 \mathrm{~dB}$ respectively. Both strategies offer up to $0.6 \mathrm{bps} / \mathrm{Hz}$ capacity enhancement compared to the equal power allocation scheme for low number of users. As expected, this performance gap closes when the number of users is increased as equal power allocation is asymptotically optimum. As seen from Fig. 3, the BOBO scheme for the $N_{t}=2$ case shows remarkable capacity gain for low to moderate number of active users. This scheme is a smooth switching from greedy power allocation, where all power is given to the best beam, and random beamforming where both beams are used with equal power each. It is obvious that this makes multiuser beamforming more robust as the above scheme, based on the threshold value $f$, is able to identify when TDMAscheduling is optimal, switching then smoothly to SDMA and offering spatial multiplexing gain. 


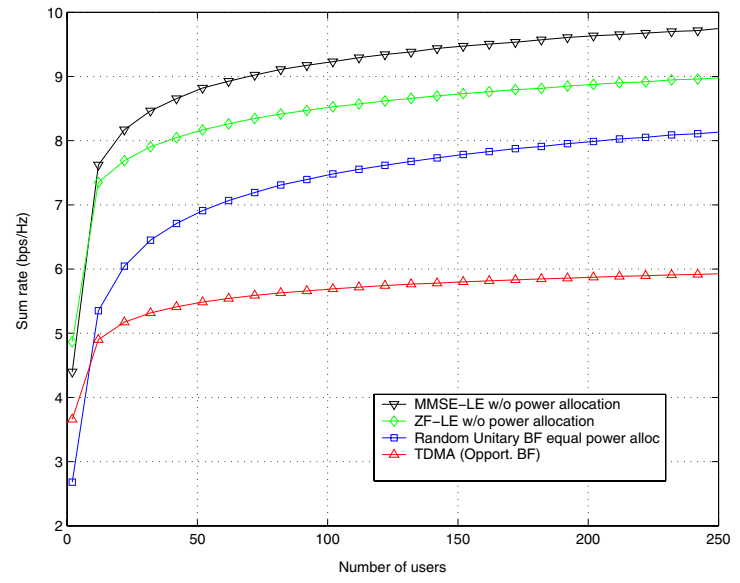

Fig. 1. Sum-rate comparison of downlink MISO schemes vs. number of users for $N_{t}=2$ and $\mathrm{SNR}=10 \mathrm{~dB}$

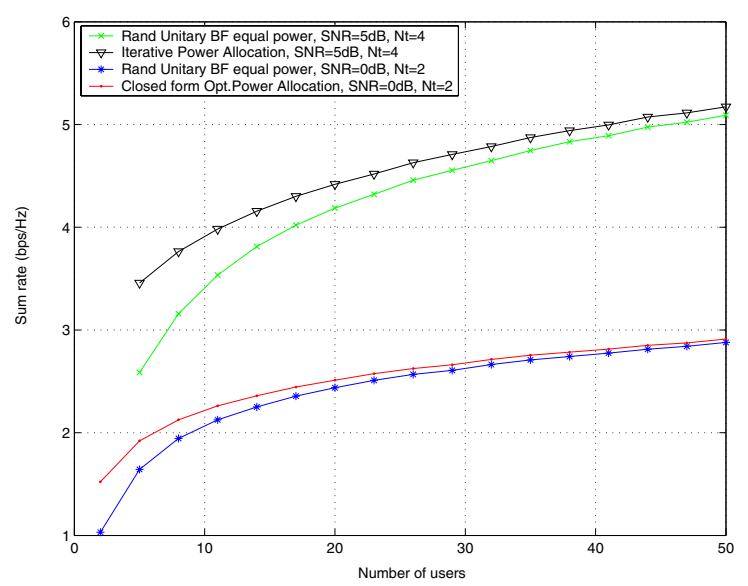

Fig. 2. Throughput vs. number of users of SIR-based power allocation strategies

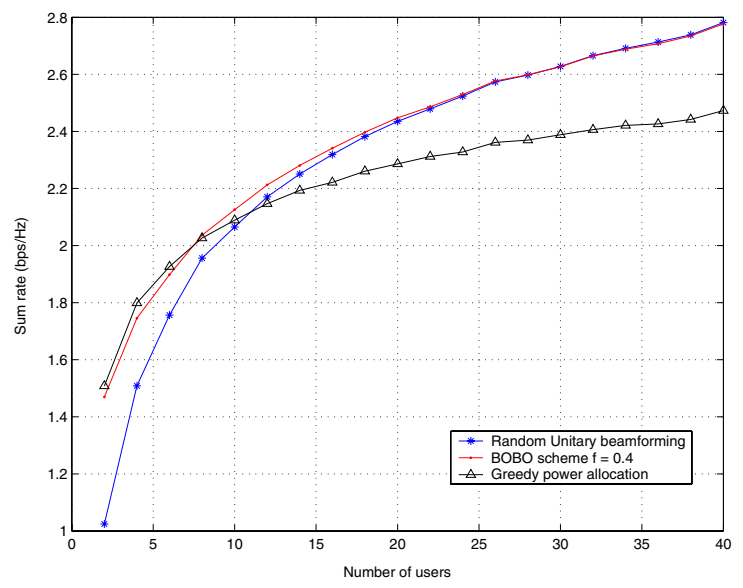

Fig. 3. Sum-rate vs. number of users of proposed 2-beam BOBO system for $\mathrm{SNR}=0 \mathrm{~dB}$

\section{CONCLUSION}

A scheme that renders opportunistic multi-user beamforming more robust in sparse networks was proposed. Depending on the level of feedback requirement, we proposed and analyzed the performance of different designs that aim to close the gap between TDMA and SDMA. We show that these schemes can offer significant capacity enhancement for low to moderate number of active users in the network.

\section{REFERENCES}

[1] M. Kobayashi, G. Caire, D. Gesbert, "Antenna diversity vs. Multiuser diversity: Quantifying the tradeoffs," in Proc. IEEE ISITA, October 2004.

[2] M. Kobayashi, G. Caire, D. Gesbert, "Transmit Diversity vs. Opportunistic Beamforming in Data Packet Mobile Downlink Transmission", IEEE Trans. on Communications, submitted, April 2005.

[3] R. Knopp, P. Humblet, "Information capacity and power control in single cell multiuser communications," in Proc. of Int. Conf. on Communications, June 1995.

[4] G. Caire, S. Shamai, "On the achievable throughput of a multi-antenna Gaussian broadcast channel," IEEE. Trans. Inform., vol. 49, no. 7, pp. 1691-1706, July 2003

[5] Z. Tu, R.S. Blum, "Multiuser diversity for a dirty paper approach," IEEE Communications Letters, vol. 7, pp. 370-372 Aug. 2003.

[6] M. Sharif, B. Hassibi, "On the capacity of MIMO broadcast channel with partial side information," IEEE. Trans. Inform., vol. 51, no. 2, pp. 506-522, February 2005.

[7] P. Viswanath, D. N. Tse, R. Laroia, "Opportunistic beamforming using dump antennas," IEEE. Trans. Inform., vol. 48, no. 6, pp. 1277-1294, June 2002.

[8] M. Kountouris, D. Gesbert, "Memory-based Opportunistic Multi-user Beamforming," submitted to IEEE Int. Symp. on Inf. Theory (ISIT), Adelaide, Australia, Sept. 2005.

[9] S. Vishwanath, N. Jindal, A. Goldsmith, "Duality, achievable rates and sum rate capacity of Gaussian MIMO broadcast channel," IEEE. Trans. Inform., vol. 49, no. 10, pp. 2658-2668, 2003.

[10] M. Sharif, B. Hassibi,, "Scaling laws of sum rate using time-sharing, DPC, and beamforming for MIMO broadcast channels," in Proc. IEEE Int. Symp. on Inf. Theory (ISIT),Chicago, IL U.S.A, July 2004.

[11] H. Boche, M. Schubert, "A General Duality Theory for Uplink and Downlink Beamforming,", in Proc. IEEE Vehicular Techn. Conf. (VTC 2002 Fall), Vancouver, Canada, Sept. 2002.

[12] M. Kountouris, D. Gesbert, T. Salzer, "Robust Opportunistic Multiuser Beamforming,", IEEE Trans. Wireless Comm., in preparation, 2005.

[13] M. Stojnic, H. Vikalo, B. Hassibi, "Rate Maximization in Multiantenna Broadcast Channels with Linear Pre-processing," in Proc. IEEE Global Telecom. Conf. (Globecom 2004), Dallas TX, USA, Nov. 2004.

[14] N. Jindal, S.A. Jafar, S. Vishwanath, A. Goldsmith, "Sum Power Iterative Water-filling for Multi-Antenna Gaussian Broadcast Channels," in Proc. Asilomar Conf. Signals, Systems, and Computers, Asilomar, CA, U.S.A, Nov. 2002. 